\title{
Challenges in Integrating Tooling and Monitoring for QoS Provisioning in SOA Systems (Keynote)
}

\author{
Adrian Mos \\ INRIA, 655 avenue de l'Europe, Montbonnot 38334, France \\ Adrian.Mos@inria.fr
}

\begin{abstract}
When addressing QoS concerns in SOA systems, some of the important challenges lie in the seamless integration of user-side tooling support with the actual execution of services and business processes. The user must be able to easily express QoS constraints and associate them with service definitions, compositions and orchestrations. The constraints must be taken into account at all levels in the increasingly complex SOA infrastructures and used to monitor and enforce SLAs, as well as to potentially drive adaptation mechanisms to improve end-to-end QoS. Conversely, QoS data generated by the execution of services and processes must be made available to the user in ways that ensure that appropriate information is presented at different levels of abstraction with the right level of granularity in order to improve understanding and future planning of service-based functionality.

These challenges can be grouped in two main areas: Driving Monitoring and Adaptation from SOA Tooling and Integrating Dynamic Monitoring Information in SOA Tooling. The first area includes means of providing simple and expressive QoS specifications within a variety of tooling options, including heavyweight editors and lightweight Web 2.0 based designers. It also includes approaches to generate appropriate monitoring artefacts (probe-specific information for provenance determination) and adaptation rules and options based on expressed QoS requirements.

The second area includes challenges in obtaining proper monitoring information at all relevant SOA levels (Enterprise Service Buses, componentplatforms such as SCA, process engines as well as lower-level grid infrastructures). It equally relates to transporting and presenting it to a large variety of editors and SOA-related visual tools (for architecture definition, process specification or service mash-ups in both heavyweight and lightweight user environments). Apart from presenting useful information to the user, QoS information must also be stored and updated in service repositories for later userreferral, as well as for use in self-adaptive environments where processes and compositions can dynamically change in order to improve QoS.

This presentation gives an overview of these challenges and presents ongoing work undertaken by INRIA for addressing them in the context of different research projects as well as the internal INRIA initiative called "galaxy". Such work includes a metamodel-based approach to transformations called the STPIntermediate Model which is part of the Eclipse SOA Tools Platform Project and that aims at unifying the SOA tooling space and relating it to monitoring data.
\end{abstract}

Keywords: SOA, quality of service (QoS), monitoring, modelling, tooling. 